\title{
References in Scholarly English and American Literary Journals Thirty Years Later: A Citation Study
}

\section{Richard Heinzkill}

This study examines 20,802 citations in 555 journal articles devoted to criticism of English and American literature published in 2003. Books are cited far more often (75.8\%) than journal articles (19.8\%). Over half of the monographs cited (55.4\%) are less than twenty years old. In general, journal articles published within the past twenty years are the most frequently cited. Literary scholars use a diversity of monographs which fall outside of the core classifications for literature; over $40 \%$ are outside. This study is compared to other citation studies of English and American literature. It concludes with observations on the use of the Web, browsing by literary researchers, and English as an interdisciplinary field.

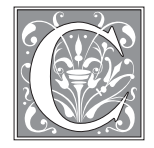

itation studies have been around for a long time. The vast majority of citation studies analyze scientific literature and are often only tangently of interest to librarians because these studies focus on issues such as questions of influence and interaction among scientists, the identification of emerging scientific disciplines and subfields, the impact of specific journals, e-journals and open access journals, as well as the methodology of citation analysis. On the other hand, librarians more often look to citation studies to inform them about the management of libraries in such areas as budgeting priorities, library services, library instruction, selecting materials for storage, and collection development. Although citation studies are not above criticism, the fact remains that "...limitations notwithstanding citations represent an auditable trail of scholarship, and citation analysis remains a useful tool for evaluation of library collections and subject literature." ${ }^{1}$ And, as always, citation studies present more reliable evidence beyond anecdotes and gut feelings about what is taking place in scholarship.

There are not many citation studies done in the humanistic disciplines, and only a few concentrate on English literature. The principal citation studies of English literature are those by Budd, Heinzkill, and Stern. ${ }^{2}$ There are other humanities citation studies that include English literature scholars; however, literature is subsumed in the totals for the whole with no breakout figures to identify activity in the various disciplines including literature.

But is it really necessary to do another study? The last citation study to deal exclusively with the field was done awhile ago (Stern, 1983), and that study concentrated on only a few areas of English and

Richard Heinzkill, retired Reference and Humanities Bibliographer, the University of Oregon; e-mail: heinzkil@uoregon.edu 
American literature. ${ }^{3}$ The presumption is that things have changed in the past thirty years! It is not necessary at this point to go into detail about changes in libraries and the impact of electronic technology on scholars; they are fairly obvious. More important, there have been many changes in the discipline of English. It has seen the full flowering of not just one, but many theories. Questions of race, gender, and class are brought to bear on texts. And going even further, questions about which works are worthy of study, especially those by women and ethnic minorities, have been raised, which in turn challenges the preeminent position of the "classics" found in anthologies of thirty years ago. These are just a few of the topics that have engaged the profession recently in their scholarly journals.

\section{Methodology}

For this analysis, articles from 42 English and American journals devoted to the scholarship of English and American literature were examined. ${ }^{4}$ Care was taken to identify journals representing a variety of editorial viewpoints. Most articles surveyed were published in 2003. (Some 2003 journal issues were not available at the time data was collected, so a few substitutions were made. ${ }^{5}$ )

From this group of scholarly journals, 555 articles dealing with literary topics were examined. Articles on art, philosophy, and history were excluded unless they referred to literary works. The articles yielded 20,802 citations (12,595 from journal articles about English literature and 8,207 from journal articles about American literature). These citations are the basis of the study.

Each article was assigned to a literary period. The imprint date of monograph citations in the article were also assigned to a time period, and the language of the monograph was identified.
Using WorldCat, the subject matter as reflected in the Library of Congress classification number of monographs published since 1800 was ascertained. The classification number of monographs classifying outside of literature parts of the PN schedule (i.e., radio, film, television, journalism), PR (English literature) and PS (American literature) were considered "nonliterary" and their frequency was tabulated to determine the use of "nonliterary" subject matter in contrast to the core literature numbers represented by PN 1-PN 1559, PR and PS.

In addition to citations to monographs, other citations were assigned to a category for journal articles or to "other" (for dissertations, newspaper articles, manuscripts, etc.). Journal articles were assigned to a literary period, date noted and the language of the article identified. Citations in the "other" category were identified as to type (e.g., dissertation, newspaper article, manuscript), and their percentage of the total number of citations was calculated.

The number of citations to non-English monographs and journal articles was tabulated by language.

\section{Principal Findings}

Books constitute 75.8 percent of all citations, journal articles 19.8 percent, and other types of material 4.4 percent. Separating the two literatures: books are 77.1 percent, journal articles 19.9 percent, and other types of material 3.0 percent in the citations pertaining to English literature. For American literature, books are 73.8 percent, journal articles 19.6 percent, and other types of material 6.6 percent.

\begin{tabular}{|l|c|c|l|}
\hline \multicolumn{4}{|c|}{ TABLE 1 } \\
Summary of Results \\
\hline \hline & \% Books & $\begin{array}{c}\text { \% Journal } \\
\text { Articles }\end{array}$ & \% Other \\
\hline English & $77.1(9,715)$ & $19.9(2,504)$ & $3.0(376)$ \\
\hline American & $73.8(6,058)$ & $19.6(1,609)$ & $6.6(540)$ \\
\hline Both literatures & $75.8(15,773)$ & $19.8(4,113)$ & $4.4(916)$ \\
\hline
\end{tabular}


(See table 1.) The two most notable differences between the literatures are that books were used slightly more in English literature than in American literature; and in American literature, the "other" category has a higher percentage because more newspaper articles were used.

\section{Age of Cited Books}

Throughout the study the publication date given in the citation was used. Often with monographs the imprint date was not the original date of publication, but a newer edition of an older work. This accounts for the use of newer imprints appearing to be higher than it might be thought for some periods. Especially in the older literary periods, citing recent editions of older works makes it appear that older works are not used as often, when in reality they are but in newer editions.

In English literary scholarship over half (51.4\%) of the books cited had imprints dated within the previous twenty years. (See table 2.) Scholarship since WWII is strongly valued inasmuch as 82 percent of the imprints fall within that time span.

Imprints of the past twenty years used for the study of American literary scholarship show a similar pattern, but with imprints from the past twenty years being more frequent than those of English literature of the same period -61.8 percent for American compared to 51.4 percent for English. And the percentage of works cited post-WWII is also higher -89.4 percent for American compared to 82.1 percent for English. (See table 3.)

\section{Characteristics of "Other" Category}

The number of citations falling into the "other" category is very small-376 citations for English literature (3.0\% of the English literature citations) although somewhat more prominent for American literature, 540 citations (6.6\% of the American literature citations). The breakdown in actual numbers for "other" is as follows:

- citations to newspapers - English (54), American (270);
- citations to dissertations-English (38), American (26);

- citations to manuscripts-English (168), American (116);

- citations to Web sites-English (39 sites, including 8 proprietary ones), American (39 sites, including 3 proprietary ones);

- citations to papers/talks - English (17), American (12);

- other citations - English (60) among which were 15 to unique copies of books usually because of handwritten marginalia, 14 to private correspondence received by author;

- other citations-American (77) - among which were 20 to conversations or interviews, 15 to audio recordings and 11 to films.

\section{Literary Subjects within Library of Congress Classification}

In English literature, the citations for imprints since 1800 are: 53.8 percent in PR (English literature), 1.3 percent in PS (American literature), and 3.4 percent in PN (literary sections only: i.e., PN 1-PN 1559) combined to make 58.5 percent of the book citations falling within the core literature sections of the classification schedule. For American literature, the citations are 42.2 percent in PS (American literature), 4.9 percent in PR (English literature), and 3.6 percent in PN (literary sections only: i.e., PN 1-PN 1559) to comprise 60.7 percent within the core literature sections of the classification schedule.

\section{Subjects Outside of Literature}

One of the unique findings of this study is the assignment of L.C. classification numbers for book citations outside of the principal literature classifications. The closest known attempt to do this is a study by Broadus in which the information needs of a broadly defined group of humanists and a subgroup of "special humanists" were analyzed. ${ }^{6}$ Although not exactly comparable, this study agrees with his observation: "The main point to be noted is the wide variety of subjects represented." ${ }^{7}$ 


\begin{tabular}{|c|c|c|c|c|c|c|c|c|c|c|c|c|c|c|c|}
\hline & 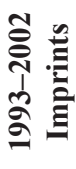 & 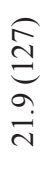 & 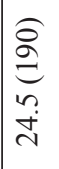 & $\begin{array}{l}\text { ñ } \\
\hat{n} \\
0 \\
\ddot{n} \\
\text { in }\end{array}$ & 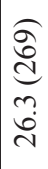 & 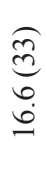 & 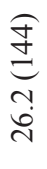 & 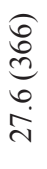 & 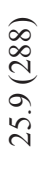 & 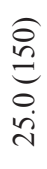 & $\begin{array}{l}\text { I } \\
\stackrel{\text { I }}{\infty} \\
\dot{0} \\
\text { di }\end{array}$ & $\begin{array}{l}\underset{n}{\approx} \\
\frac{0}{\infty}\end{array}$ & $\begin{array}{l}\hat{8} \\
\stackrel{2}{2} \\
\stackrel{2}{1}\end{array}$ & 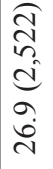 & 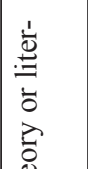 \\
\hline & 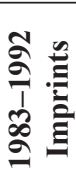 & 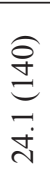 & 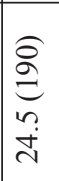 & $\begin{array}{l}\text { f } \\
\text { in } \\
0 \\
\ddot{d} \\
\text { in }\end{array}$ & 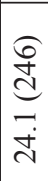 & 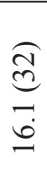 & 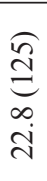 & 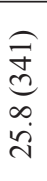 & 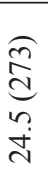 & 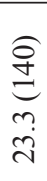 & 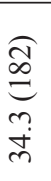 & 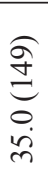 & $\begin{array}{l}\tilde{\partial} \\
\dot{\infty} \\
\dot{v} \\
\tilde{d}\end{array}$ & 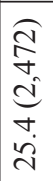 & 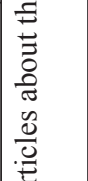 \\
\hline 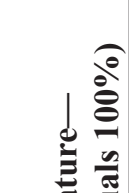 & 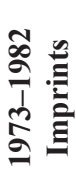 & 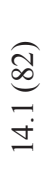 & 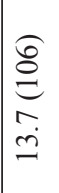 & 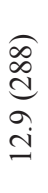 & 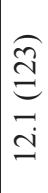 & 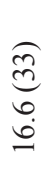 & 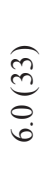 & 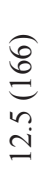 & 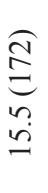 & \begin{tabular}{l}
6 \\
\multirow{2}{*}{} \\
$i$
\end{tabular} & $\begin{array}{l}\hat{\sigma} \\
\stackrel{\infty}{0} \\
\ddot{n}\end{array}$ & $\begin{array}{l}\overparen{E} \\
0 \\
0\end{array}$ & $\begin{array}{l}\underset{\mathfrak{I}}{\infty} \\
\stackrel{\Xi}{\Xi}\end{array}$ & 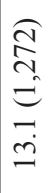 & 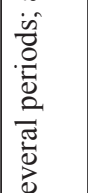 \\
\hline 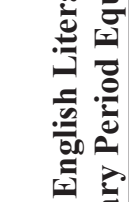 & 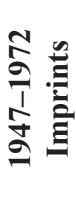 & 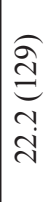 & 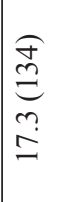 & 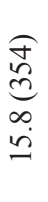 & $\begin{array}{l}\frac{\widehat{I}}{d} \\
\frac{\sim}{d}\end{array}$ & 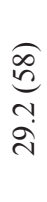 & $\begin{array}{l}\underset{\Xi}{\sigma} \\
\underset{\Xi}{\sigma} \\
\dot{0}\end{array}$ & 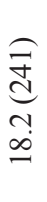 & 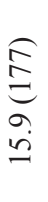 & 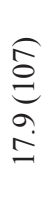 & $\begin{array}{l}\widehat{\sigma} \\
\infty \\
\infty \\
0 \\
0\end{array}$ & $\begin{array}{l}\text { } \\
\theta \\
\exists \\
\dot{J}\end{array}$ & $\begin{array}{l}\hat{\sigma} \\
\text { c } \\
\vdots \\
0\end{array}$ & 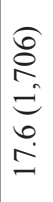 & 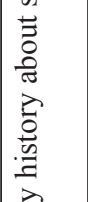 \\
\hline 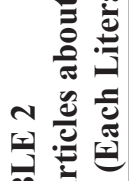 & 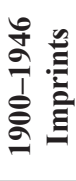 & 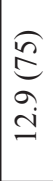 & $\begin{array}{l}\stackrel{E}{a} \\
a\end{array}$ & 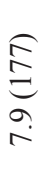 & $\begin{array}{l}\underset{\overrightarrow{0}}{0} \\
0 \\
\dot{m}\end{array}$ & 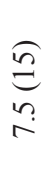 & 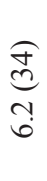 & 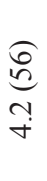 & $\begin{array}{l}\text { } \\
\stackrel{\Xi}{=} \\
=\end{array}$ & 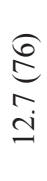 & 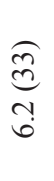 & $\underset{m}{\stackrel{\overbrace{}}{\Xi}}$ & 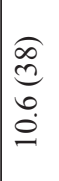 & 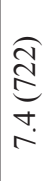 & 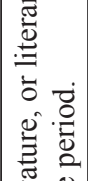 \\
\hline ㅂ. & 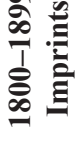 & $\begin{array}{l}\underset{\mathfrak{d}}{d} \\
? \\
+\end{array}$ & 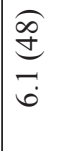 & 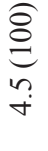 & 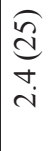 & $\begin{array}{l}\overparen{d} \\
0 \\
0\end{array}$ & 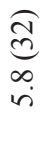 & 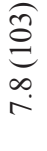 & $\underset{\substack{\infty \\
\infty \\
\infty}}{\substack{\infty \\
\infty}}$ & $\begin{array}{l}\underset{\partial}{+} \\
\underset{\infty}{\infty}\end{array}$ & 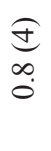 & $\mid$ & $\begin{array}{l}\underset{g}{ \pm} \\
n \\
=\end{array}$ & \begin{tabular}{l}
$\underset{\infty}{\infty}$ \\
\multirow{n}{c}{} \\
$\dot{v}$ \\
$\dot{n}$
\end{tabular} & 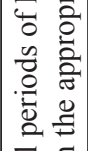 \\
\hline 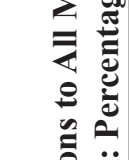 & 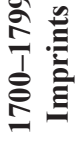 & 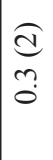 & $\begin{array}{l}\stackrel{\Xi}{0} \\
\overrightarrow{0}\end{array}$ & $\begin{array}{l}\stackrel{\tilde{2}}{\rightleftharpoons} \\
\stackrel{0}{0}\end{array}$ & $\underset{\substack{\infty \\
0}}{\stackrel{\infty}{0}}$ & $\begin{array}{l}\underset{\infty}{\infty} \\
\underset{\forall}{+}\end{array}$ & $\begin{array}{l}\text { E} \\
\text { à }\end{array}$ & $\begin{array}{l}\widehat{\vec{\sigma}} \\
\stackrel{\sigma}{\sigma} \\
\text { ले }\end{array}$ & $\underset{m}{\tilde{n}}$ & $\begin{array}{l}\overparen{\Xi} \\
\stackrel{\Xi}{\sigma}\end{array}$ & $\mid$ & $\mid$ & 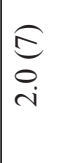 & 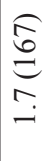 & $\begin{array}{ll}0 & \text { ] } \\
0 & 0 \\
0 & 0 \\
0 & 0 \\
0 & 0 \\
0 & 0 \\
0 & 0 \\
0 & 0\end{array}$ \\
\hline 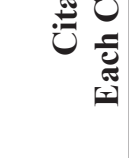 & 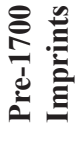 & $\mid$ & 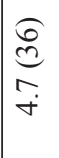 & $\begin{array}{l}6 \\
\stackrel{6}{n} \\
\stackrel{0}{r}\end{array}$ & \begin{tabular}{l}
\multirow{2}{0}{} \\
$=$ \\
0
\end{tabular} & $\begin{array}{l}\underset{\infty}{=} \\
0 \\
a\end{array}$ & $\begin{array}{l}\hat{\sigma} \\
\cong\end{array}$ & | & | & $\mid$ & $\mid$ & $\mid$ & $\begin{array}{l}\underset{ \pm}{ \pm} \\
= \\
=\end{array}$ & 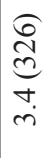 & 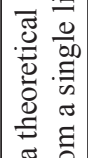 \\
\hline & 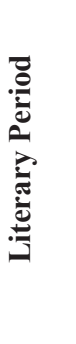 & 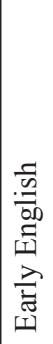 & 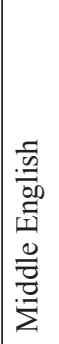 & 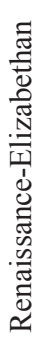 & 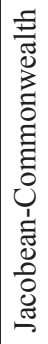 & 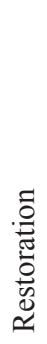 & 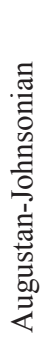 & 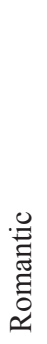 & 蔦 & 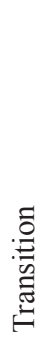 & 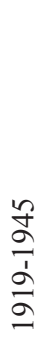 & \begin{tabular}{l}
1 \\
8 \\
0 \\
1 \\
$b$ \\
\multirow{2}{1}{}
\end{tabular} & 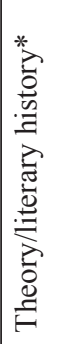 & $\frac{n}{\frac{\pi}{\pi}}$ & 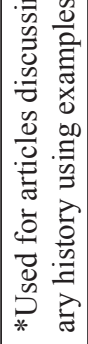 \\
\hline
\end{tabular}




\begin{tabular}{|c|c|c|c|c|c|c|c|c|c|}
\hline :气 & 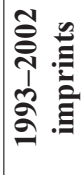 & $\begin{array}{l}\widehat{a} \\
\hat{a} \\
0 \\
\dot{0} \\
\text { in }\end{array}$ & 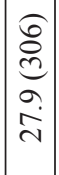 & $\begin{array}{c}\widehat{\vec{n}} \\
\tilde{n} \\
n \\
\infty \\
\sim \\
\end{array}$ & 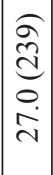 & $\begin{array}{l}\widehat{\sigma} \\
\dot{f} \\
0 \\
0 \\
0 \\
\dot{y}\end{array}$ & 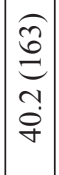 & 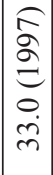 & 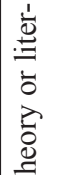 \\
\hline 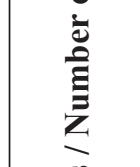 & 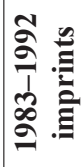 & 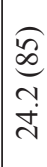 & 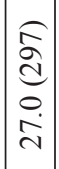 & 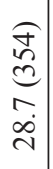 & 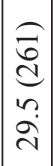 & 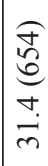 & 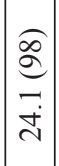 & 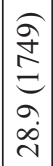 & $\begin{array}{l}0 \\
0 \\
0 \\
0 \\
0 \\
0 \\
0.0 \\
0.0 \\
0\end{array}$ \\
\hline 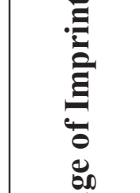 & 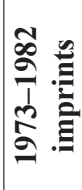 & $\begin{array}{l}\infty \\
0 \\
0 \\
n \\
0 \\
-1\end{array}$ & 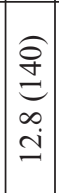 & 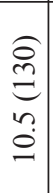 & 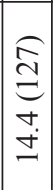 & 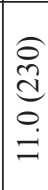 & 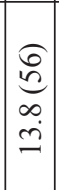 & 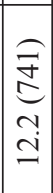 & 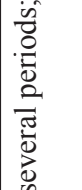 \\
\hline 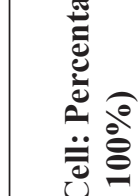 & 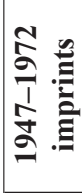 & 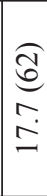 & $\begin{array}{l}\infty \\
\infty \\
= \\
= \\
=\end{array}$ & 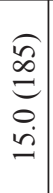 & 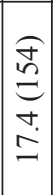 & 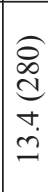 & $\begin{array}{c}\hat{\theta} \\
\dot{8} \\
\infty \\
\dot{ \pm}\end{array}$ & 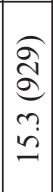 & 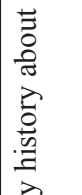 \\
\hline m & 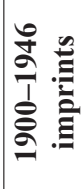 & 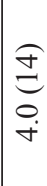 & 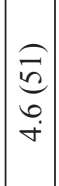 & $\begin{array}{l}\stackrel{\overbrace{}}{\stackrel{g}{\Xi}} \\
\underset{\sigma}{ \pm} \\
=\end{array}$ & $\begin{array}{l}\tilde{2} \\
\hat{\sigma} \\
\mathfrak{0} \\
0\end{array}$ & $\begin{array}{l}\underset{\Xi}{\Xi} \\
n \\
m\end{array}$ & 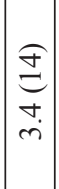 & 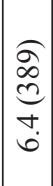 & 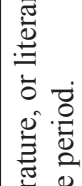 \\
\hline 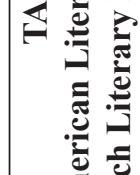 & 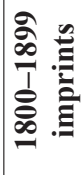 & 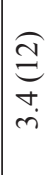 & $\begin{array}{l}\stackrel{f}{0} \\
\stackrel{\Xi}{n} \\
a\end{array}$ & 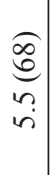 & $\begin{array}{l}\hat{\infty} \\
\vdots \\
0 \\
0\end{array}$ & $\begin{array}{l}\vec{\Xi} \\
\overrightarrow{\dot{\theta}}\end{array}$ & $\begin{array}{l}\tilde{m} \\
\stackrel{\sim}{\sim} \\
\tilde{m}\end{array} \mid$ & 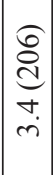 & 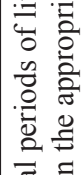 \\
\hline 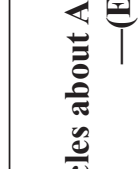 & 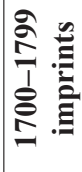 & $\begin{array}{l}\underset{\sigma}{\sigma} \\
\infty \\
i \\
\text { i }\end{array}$ & $\begin{array}{l}\overparen{\Xi} \\
\Xi \\
\beth\end{array}$ & $\begin{array}{c}\text { d } \\
\text { ñ } \\
0\end{array}$ & $\begin{array}{l}Ð \\
\ddots \\
0\end{array}$ & $\begin{array}{l}\overparen{\Xi} \\
\overrightarrow{\dot{\theta}}\end{array}$ & $\begin{array}{l}\overparen{d} \\
n \\
0\end{array}$ & 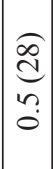 & 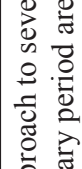 \\
\hline $\begin{array}{l}\sum \\
. \Xi \\
\frac{\vdots}{a} \\
\frac{a}{\pi}\end{array}$ & 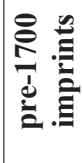 & $\begin{array}{l}\overparen{\sigma} \\
\underset{v}{\Xi} \\
\dot{v}\end{array}$ & 1 & $\mid$ & $\mid$ & $\mid$ & $\mid$ & 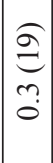 & 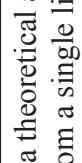 \\
\hline 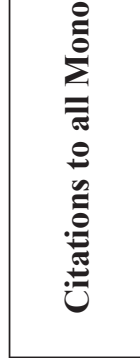 & 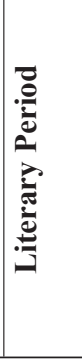 & 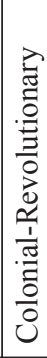 & 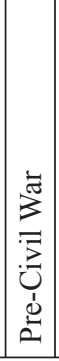 & $\begin{array}{l}\infty \\
\sigma \\
\frac{1}{1} \\
\infty \\
\infty\end{array}$ & $\begin{array}{l}\frac{2}{2} \\
2 \\
1 \\
a \\
a \\
2\end{array}$ & 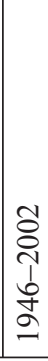 & 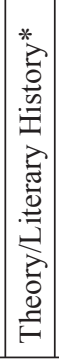 & 足 & 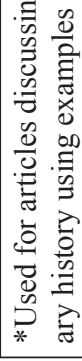 \\
\hline
\end{tabular}

Exactly how wide the subject range is becomes apparent in table 4 where monographs published since 1800 and classifying outside of PN literary sections (i.e., in the radio, film, television and journalism sections), outside of PR (English literature) and outside of PS (American literature) are identified by broad su bject numbers. ${ }^{8}$ In addition to the expected interest in philosophy, religion, history, and sexuality, the subjects consulted run the gamut from $\mathrm{A}$ to $\mathrm{Z}$ in the $\mathrm{Li}$ brary of Congress subject scheme. More significant is the large portion of these "nonliterary" citationsother subject monographs constituted 41.5 percent $(3,825$ titles) of the total of the monographs cited from English literature articles and 49.3 percent (2,963 titles) from American literature articles were outside of the literature core.

No claim is made that citations tabulated in table 4 are all unique. For instance, the percentages include multiple citations to the works of Pierre Bourdieu, Judith Butler, Jacques Derrida, Michel Foucault, and Fredric Jameson. Considering the emphasis on theory their works do not show up with as much regularity as was expected. It is estimated multiple citations to these authors do not significantly skew the figures. It was further observed during the course of doing this study there were not any works that could be considered to be heavily cited. This observation corresponds with another literary citation study, which states: "Few titles are cited with any frequency." 9

\section{Characteristics of Citations to Journal Articles}

Summary table 3 indicates journal articles account for 21.5 percent of the citations. 


\section{TABLE 4}

\section{Monographs (Published 1800-2002) Outside of Literature Classifications}

\begin{tabular}{|c|c|c|}
\hline L.C. Classification & $\begin{array}{c}\text { \% Appeared } \\
\text { in Articles } \\
\text { about } \\
\text { English } \\
\text { Literature }\end{array}$ & $\begin{array}{c}\text { \% Appeared } \\
\text { in Articles } \\
\text { about } \\
\text { American } \\
\text { Literature }\end{array}$ \\
\hline A - some collected works, encyclopedias, museums & 0.5 & 0.3 \\
\hline B - philosophy & 5.5 & 3.5 \\
\hline BF - psychology & 3.8 & 3.9 \\
\hline $\mathrm{BH}$ - aesthetics & 0.8 & 0.5 \\
\hline $\mathrm{BL}-\mathrm{BQ}$ - non-Christian religions & 1.0 & 1.1 \\
\hline BR-BS - Christianity, Bible & 5.8 & 1.1 \\
\hline BT-BV - theology & 5.8 & 2.6 \\
\hline Other B classifications in philosophy & 1.4 & 4.8 \\
\hline $\begin{array}{l}\mathrm{C} \text { - history of civilization, diplomatics, general } \\
\text { biography }\end{array}$ & 1.3 & 1.0 \\
\hline DA - history of British Isles & 12.4 & 0.7 \\
\hline Other D classifications — European history & 4.6 & 2.9 \\
\hline $\begin{array}{l}\mathrm{E} \text { - American history, including experience of ethnic } \\
\text { groups in USA }\end{array}$ & 1.2 & 23.5 \\
\hline $\begin{array}{l}\text { F - regional US history, history of Northern and } \\
\text { Southern hemispheres }\end{array}$ & 0.7 & 7.8 \\
\hline GN - anthropology & 0.8 & 1.9 \\
\hline Other G classifications in geography, folklore & 1.5 & 1.5 \\
\hline H-HA - general social studies, statistics & 0.3 & 0.1 \\
\hline $\mathrm{HB}$ - economic theory & 0.9 & 0.5 \\
\hline HC-HD - economic history, economic conditions & 1.5 & 2.1 \\
\hline HE-HG - commerce and finance & 0.4 & 0.7 \\
\hline HM - sociology & 1.0 & 1.7 \\
\hline $\mathrm{HN}$ - social history, social problems, social reform & 1.1 & 0.5 \\
\hline $\mathrm{HQ}$ — family, marriage, women, sexuality & 5.3 & 6.0 \\
\hline HS-HT - societies, social classes, slavery & 0.8 & 0.9 \\
\hline HV - public welfare, criminology & 1.4 & 1.3 \\
\hline $\mathrm{HX}$ - socialism, communism & 0.7 & 1.8 \\
\hline $\mathrm{JC}$ - political theory & 2.0 & 1.2 \\
\hline JK - U.S. political institutions & - & 0.6 \\
\hline $\mathrm{JN}$ - constitutional history & 1.0 & $<0.1$ \\
\hline $\mathrm{JV}$ - colonization & 0.5 & 0.1 \\
\hline Other $\mathrm{J}$ classifications in political science & 0.7 & 0.5 \\
\hline $\mathrm{K}$ - law & 1.7 & 1.6 \\
\hline
\end{tabular}




\begin{tabular}{|l|c|c|}
\hline \multicolumn{3}{|c|}{ TABLE 4 } \\
Monographs (Published 1800-2002) Outside of Literature Classifications \\
\hline \hline L.C. Classification & $\begin{array}{c}\text { \% Appeared } \\
\text { in Articles } \\
\text { about } \\
\text { English } \\
\text { Literature }\end{array}$ & $\begin{array}{c}\text { \% Appeared } \\
\text { in Articles } \\
\text { about } \\
\text { American } \\
\text { Literature }\end{array}$ \\
\hline L - education & 1.2 & 1.1 \\
\hline M - music, musicology & 0.3 & 3.0 \\
\hline N - NC - art general, architecture, sculpture & 1.0 & 1.0 \\
\hline ND-NK - painting, printmaking, decorative arts & 1.3 & 0.3 \\
\hline NX - special arts topics & 0.9 & 1.4 \\
\hline P - linguistics & 2.4 & 1.1 \\
\hline PA - Greek and Latin literature & 3.9 & 0.5 \\
\hline PB-PD - Celtic, Germanic linguistics & 0.8 & 0.1 \\
\hline PE - English linguistics & 4.3 & 2.3 \\
\hline PG-PM - Slavic, Native American literatures & 0.1 & 0.6 \\
\hline PN - only theater, film, television, journalism & 5.6 & 5.0 \\
\hline PQ - French literature & 2.8 & 1.6 \\
\hline PQ - Spanish literature & 0.8 & 2.0 \\
\hline PQ - Italian literature & 0.2 & 0.5 \\
\hline PT - German literature & 0.4 & 0.5 \\
\hline Q-QP - science & 1.7 & 1.5 \\
\hline RC - psychiatry & 1.5 & 1.3 \\
\hline Other R classifications in medicine & 1.0 & 0.7 \\
\hline S - science & 0.1 & 0.3 \\
\hline T - technology & 1.5 & 0.8 \\
\hline U-V - military & 0.2 & $<0.1$ \\
\hline Z - book history, industry & 1.1 & 0.6 \\
\hline Z - libraries, manuscript catalogs & 0.5 \\
\hline
\end{tabular}

In general, articles published within the past twenty years are the most frequently cited.

In English literature, the work on modern literature (i.e., belles-lettres since 1919) relies most heavily on journals published in the last ten years -53.0 percent of journal citations for the literary period 1919-1945 were to articles published in the last ten years, and for the literary period 1946-2002, 49.9 percent of articles published were published in the last ten years. If another ten years are added (i.e., journals published in the last twenty years), every literary period except Early English and to a lesser degree Middle English and Transition favored articles published 1983-2002. ${ }^{10}$

In American literature, the journal articles published during the past ten years accounted for 40.2 percent of the journal articles cited in scholarship about the 1919-1945 period; 50.2 percent of the articles for the period 1946-2002; and 


\begin{tabular}{|l|c|c|c|}
\hline \multicolumn{4}{|c|}{ TABLE 5 } \\
\hline & $\begin{array}{c}\text { \% } \\
\text { Books }\end{array}$ & $\begin{array}{c}\text { \% } \\
\text { Articles }\end{array}$ & $\begin{array}{c}\text { \% } \\
\text { Other }\end{array}$ \\
\hline \hline $\begin{array}{l}\text { Budd (1986) } \\
\text { (American literature) }\end{array}$ & 64.0 & 23.0 & 12.9 \\
\hline $\begin{array}{l}\text { Gleaves (1960) } \\
\text { (American literature) }\end{array}$ & 49.9 & 24.7 & 25.4 \\
\hline $\begin{array}{l}\text { Heinzkill (1980) } \\
\text { (English literature) }\end{array}$ & 74.9 & 19.9 & 5.2 \\
\hline $\begin{array}{l}\text { Heinzkill (this study) } \\
\text { (English literature) }\end{array}$ & 77.1 & 19.9 & 3.0 \\
\hline $\begin{array}{l}\text { Heinzkill (this study) } \\
\text { (American literature) }\end{array}$ & 73.8 & 19.6 & 6.6 \\
\hline $\begin{array}{l}\text { Stern (1983) } \\
\text { (3 English and } \\
\text { American authors) }\end{array}$ & 82.7 & 15.1 & 2.2 \\
\hline $\begin{array}{l}\text { Stern (1983) } \\
\text { (3 literary movements) }\end{array}$ & 78.8 & 16.5 & 4.6 \\
\hline
\end{tabular}

variety of languages were represented (Chinese, Danish, Dutch, Icelandic, Old Norse, Polish, Slovak, and Spanish being among them). Journal articles yielded 61 non-English citations. (A single article, a reception study on Dickens in Italy, accounted for half of these citations.) Other languages represented among citations to journal articles were: French (13 citations), German (11 citations) and Spanish (1 citation).

The articles on American literature used even fewer nonEnglish monograph sources-29 French, 11 Spanish, 5 German, and 1 Italian for a total of 46 citations. Journal articles were almost completely reliant on English language material as there were only 7 non-English citations -6 in French, 1 in German.

54.9 percent for literary theory and history category. But the past ten years are not as important for the other literary periods-Colonial, 22.1 percent published during the past ten years; pre-Civil War, 28.1 percent, and 1861-1918, 31.3 percent. If this time span is enlarged to the past twenty years, every American literary period (except the pre-Civil war period) shows that at least 50 percent of the articles cited were published between 1983 and 2002. ${ }^{11}$

\section{Appearance of Non-English Material}

Although obviously important to researchers who used non-English material, these materials are not a significant factor in the scholarship-witness the low number of citations to non-English monographs: in the English literature section (266 citations) and in the American literature section (46 citations).

In English literature the four non-English languages most frequently cited were French (100 citations), Latin (75 citations), German (43 citations), and Italian (26 citations). Even though no other language garnered more than five citations, a

\section{Comparison with Other Studies}

The title of this study includes the phrase "thirty years later," which refers to the use of articles published in 2003 (with a few exceptions). This is roughly thirty years after the sources for my 1980 article, which used articles published 1972-1974. ${ }^{12}$ Unlike the 1980 article, which looked at only British literature, the present study includes American literature. ${ }^{13}$

Table 5 compares book and journal citations of several other studies. When comparing the studies, one should be aware they were done using different methodologies, including different sources. Nevertheless, they do show that, no matter what the methodology, over the years literary scholars have relied more heavily on books than journal articles and continue to do so.

The comparison with results of the citations to English literature in the 1980 article-the proportion of books/articles/other is not all that different from 1980 -books $(77.1 \%$ to $74.9 \%$ in 1980$)$; articles are exactly the same $(19.9 \%$ and $19.9 \%$ in 1980); and the "other" category is 
down $(3.0 \%$ to $5.2 \%$ in 1980$)$. The number of citations used in the present study is higher (20,802 to 9,556 in 1980) and comes from a wider range of journals than the 15 journals the 1980 study examined.

The citing of monographs published during the most recent ten-year period of each study is almost exactly the same. In the 1980 article, 25.89 percent of the monographs were published within ten years immediately preceding the study; in the present study, 26.9 percent of the monographs were published within the ten years preceding the study. However, the dates of the journal literature show an increase in the use of recently published journal articles. In 1980, the journal articles published for the most recent ten-year period preceding the study constituted 38.56 percent of all of the journal articles; in this study, the ten years preceding the study were 47.0 percent of all journal articles.

The most dramatic difference between the 1980 study and this study is in the use of non-English material. The articles in this study used over 60 percent more book citations than in 1980, yet the actual number of non-English citations dropped from 795 in 1980 to 376 . And the drop in non-English articles was even more precipitous: 106 in 1980 to 61 .

The age of materials is harder to compare because the various studies gathered data in different time increments. Budd's study of American literature done with 1981 sources offers some comparable figures. ${ }^{14}$ In his study, the percentage of books with imprints for the previous decade is 29.4 percent, which is almost identical to this study (29.1\%). If the time frame in Budd's study is extended to imprints for the twenty-year period preceding his study, he found 53 percent compared to this study's 57.4 percent. For older material, the difference is greater. In Budd's study, 29.2 percent of titles were 50 years or older. The present study finds only 10.6 percent of the titles older than 55 years.

The only other study that compares the age of material in English literary studies is the one by Stern (1981)..${ }^{15}$ Most of her data is not directly comparable. She states that for the selected literary movements she found 30 percent of "published references are ten years old or less," and, for the three British and American authors she followed, 21.6 percent of "published references are ten years old or less." This would indicate that the majority of items used are older than ten years, which corresponds with the findings here. ${ }^{16}$ However, Broadus' look at "those scholars working more strictly in the humanities" finds a higher percentage of requests for titles less than 18 years old $(63.9 \%)$, and 81.2 percent of the titles were published within the previous 34 years. ${ }^{17}$

Broadus, observing the interlibrary loan patterns of humanists at a research center, divided requests from "those scholars working more strictly in the humanities" into broad subject areas (i.e., general, history, humanities, sciences, and social sciences); 43.4 percent of the requests were for items outside of what he defines as the humanities. ${ }^{18}$ He does give percentages for various groupings of Library of Congress classification numbers, but the groupings vary from those in table $4 .^{19}$

This study is in line with other observations that humanists and scientists use distinctly different types of library resources. The ratio of use between books and journals in the sciences is almost the reverse of that found in the humanities. "The bulk of scientific literature which is published throughout the world appears in serials ... [In 1956] in eight major scientific subject fields the percentage of citations to serials in relation to the total number of citations to books and all serials was in the order of 85.2 percent." ${ }^{20} \mathrm{Sev}$ eral years later Rosalind Walcott "states that the cited geoscience literature is predominantly serial in form, (serials were $75-86 \%$ of citations, monographs were approximately $12-22 \%$ of citations)." 21 She also found the range of percentages of serials in the research literature of the biological sciences is from 80 percent to 
93 percent. ${ }^{22}$ Claudia Lascar and Loren Mendelsohn give a figure that ranks serials even higher-95.1 percent of the citations in the literature of structural biology are to journals. ${ }^{23}$ They further state: "This high proportion is consistent with established theory that the majority of scholarly communication in the scientific community takes place in the journals," which is practically the opposite situation in the humanities where books are more highly valued than journal articles. ${ }^{24}$

\section{Use of the Web and Electronic Resources}

As Palmer and Neumann found in 1995-1997, humanists are not adverse to using electronic technology for personal communication, to manage their research, to scan documents, and to write, but as of that time period "the digital is secondary to the physical in the actual use of information sources, suggesting that full-text materials are not yet aligned with scholarly work practices." ${ }^{25}$ This study agrees with this conclusion-with a few caveats, however. First of all, some studies group historians in with literary scholars. One would expect historians to be interested in more primary documents, which are being digitized at a rapid clip. Even though there are almost no cites to digitized literary texts, it is probable they are used in the research process by literature scholars but not cited. ${ }^{26}$ One reason may be that most literary e-texts that tend to be older, out of copyright texts, have an electronic "find" capability that locates keywords instantaneously within a text. Once the location of a passage is known in the electronic version, it can then easily be located in a printed newer scholarly edition. The passage is then cited from the paper edition, which citation carries with it more cachet than a citation to an electronic version. This scenario may account for the almost complete lack of citations to primary texts in electronic format among the articles surveyed in this study.

Humanistic scholars may not be strangers to the Web, but there was little evidence (at least as of 2003) of much activity on the Web in their published articles. There were a few referrals to e-mails (included in "other"). There were fewer than two dozen references to e-journals - the most frequently cited being Postmodern Culture. Only a couple of articles were acknowledged to have been accessed electronically. There may be more access to electronic journals than the statistics would indicate, especially to the journals offered through Project Muse and JSTOR. These electronic databases display the text with page numbers as they appear in the printed copy, so a citation need not indicate the electronic version was read, and the articles can be cited as if they had been read in the paper version.

As the discussion above of "other" material states, there were eleven mentions of proprietary databases (i.e., databases that require an institutional subscription). Several citations were to LION (LIteratureONline from Chadwyck-Healey). Citations to other kinds of Web sites were very infrequent, a total of 67 references in both the English and American literature sections to free (i.e., nonproprietary) Web sites. One can theorize on the reasons for this: there is little on free Web sites that is worth citing, or perhaps free Web sites are perceived to be so unstable that it is a disservice to cite them lest readers in years hence search for the site in vain.

\section{Use of Browsing}

It is well known that browsing is an important part of the research process for humanist scholars. One way to get started browsing is to use catalog and database search results as the jumping-,off point to identify shelves where promisingsounding titles are to ascertain if items are indeed relevant and worth further attention. ${ }^{27}$ Even after having read an item, a scholar may decide not to incorporate it into the discussion, in which case a read item would not merit a footnote. Although unaware of any research confirming the ratio of items consulted 
to items cited, I suggest that for every footnote there are 3-5 items that have been perused but are not cited for various reasons (e.g., looked promising, but turns out not to be relevant, contribution to the final scholarly article is too slight to merit a footnote). If this is the case, the number of monographs consulted will be much higher than the figures in this study, which means there is more activity at shelves outside the literature sections than one might suspect. ${ }^{28}$

\section{English Literature-An Interdisciplinary Study?}

The percentages in table 4 (monographs outside of literature) are small for most call number ranges, but accumulate to represent a substantial amount of citations outside of literary -41.5 percent for English literature and 49.3 percent for American literature. How did scholars discover the titles that fall outside of their primary field of study - through a library catalog, interaction with librarians, databases furnished by the library, browsing at the shelf, footnote chasing, conversations with colleagues? These are a few of the avenues that come to mind. But a scholar in terra incognito experiences these avenues in a different way than when researching in his or her home territory. One study by Palmer and Neumann touches on this. They set out to investigate among other things "How do researchers find out and use information from areas outside their core domain?"29 Because theirs is an ethnographic study following the work habits of those doing scholarship, their answers are more general than specific. Use of the library, databases, special collections, and so on are identified, but techniques scholars used to mine these resources are not spelled out. Their subjects are faculty from many academic departments within the humanities who were selected for the interdisciplinary focus of their work; thus, the findings pertain to scholarship done in an interdisciplinary mode. This interdisciplinary sense is somewhat dif- ferent from an English literary scholar using findings from other disciplines to flesh out background in a literary paper, to discuss a philosophical point, or to put a piece of literature into a broader context. Using works outside literary fields does not necessarily make a literary study interdisciplinary especially interdisciplinary as defined by Giles Gunn - “To bring two or more disciplines into significant interaction with one another ..." [italics mine $].{ }^{30}$ Whatever the interest above and beyond the strictly literary is called, it is an important aspect of information seeking by literature researchers and needs further investigation. ${ }^{31}$

\section{Implications for Literature Selectors}

Developing a strong literature collection is only one aspect of the work of the literature selectors. They have to become informed about the directions the interests of their faculty and students are taking. As this study shows, their interests lie in many different areas outside the literary classifications (PN, PR, and PS) - over 40 percent of the citations to monographs published since 1800 are to material outside of the literature sections of the library. (And this may be a conservative figure because, as is well known, all items consulted are not cited.) Additional emphasis on the importance of this comes from Palmer and Neumann: "Information that is gathered from outside domains often requires additional resources before it can be understood and used." 32 Because resources consulted in today's literature studies run the full spectrum of the L.C. classification schedule, there are also implications for library instruction. It is important for literary scholars, in addition to being acquainted specifically with the literature resources, to be able to find their way around the other resources the library has to offer.

\section{Conclusion}

Despite changes in libraries, in the profession, and in the way scholarship is being done in a computerized world, 
when this study is compared to previous citation studies there is little evidence that the mix of library resources being used for English literary scholarship has changed dramatically. Books, especially those published since WWII, continue to be the favored resource. This long period of usefulness should be an incentive to continually assess the collection for lacunae and then engage in some retrospective book selection. With half of the journal citations to articles published since 1946, continued access to older journals is important whether they are on the shelf or provided electronically. Although non-English resources were never a significant part of English literary scholarship, their use has declined even further. One unique finding is that over forty percent of the citations were outside of the literature range in the Library of
Congress number scheme. The range of "nonliterary" titles extends beyond the books in religion, philosophy, and history sections to practically every call number area. The high use of "nonliterary" material calls for the literature selector to be aware of the scholarly interests of faculty and students they work with in order to monitor the adequacy (and if necessary select) in "nonliterary" subject areas. Furthermore, in addition to instruction aimed specifically at locating and using literature resources, students need familiarity with general principles of searching library catalogs and databases in a variety of disciplines. And finally, although this citation study is only one indicator in the information-seeking behavior of a group of humanistic scholars, it contributes to a better understanding of library usage by a group that are heavy library users.

\section{Notes}

1. John Laurence Kelland and Arthur P. Young, "Citation Patterns and Library Use," in Encyclopedia of Library and Information Science, ed. Allen Kent (New York: Marcel Dekker, 1998), vol. $61,62$.

2. John Budd, "Characteristics of Written Scholarship in American Literature: A Citation Study," Library and Information Science Research 8 (1986): 189-211; Richard Heinzkill, "Characteristics of References in Selected Scholarly English Literary Journals," Library Quarterly 50 (1980): 352-65; Madeleine Stern, "Characteristics of the Literature of Literary Scholarship," College E Research Libraries 44 (1983): 199-209.

3. See note 2 above.

4. Sources are relevant articles published for the volume year 2003 (unless otherwise noted). Journal titles and number of articles analyzed are: African American Review (35), American Literary History (32), American Literary Realism (14), American Literature (23) Callaloo (19 in vol. 25, no. 4, [2002]; vol. 26, no. 1, 2, 3), Contemporary Literature (15), Early American Literature (14), English Literature in Transition (12), Essays in Criticism (13), Exemplaria (9), Isle (8), Journal of English and Germanic Philology (10), Legacy (9), Modern Fiction Studies (25), Modern Language Quarterly (11), Modern Language Review (9), Neophilologus (9), New England Quarterly (7), Papers on Language and Literature (18), PMLA (15), Representations (11), Review of English Studies (22 in vol. 52 [2001]), Shakespeare Quarterly (11), Southern Literary Journal (16), Speculum (5), Studies in American Fiction (10), Studies in English Literature (36), Studies in Romanticism (15), Texas Studies in Literature and Language (16), Tulsa Studies in Women's Literature (10), Yale Journal of Criticism (7), and randomly selected articles from: CLA Journal (4), Eighteenth Century Fiction (5), ELH (31), English Language Notes (8), English Literary Renaissance (6), Medium Aevum (6), Neuphilologische Mitteilungen (3), Studies in Philology (7), Twentieth Century Literature (8), Victorian Literature and Culture (7), Victorian Studies (4).

5. See note 4 above.

6. Robert N. Broadus, "Information Needs of Humanities Scholars: A Study of Requests Made at the National Humanities Center," Library and Information Science Research 9 (1987): 113-29. His grouping of L.C. classification categories is different from the grouping done for this study. He also includes older titles, which this study does not.

7. Ibid., 117.

8. The year 1800 is used as the cutoff based on the presumption that volumes from earlier centuries are in rare book collections where it is not usually possible for the scholar to browse 


\section{References in Scholarly English and American Literary Journals 153}

the shelf; thus, the figures here reflect activity at shelves throughout the library. This is important inasmuch as browsing is of prime importance to humanists, especially when they leave their core subject area; see Carole L. Palmer and Laura J. Neumann, "The Information Work of Interdisciplinary Humanities Scholars: Exploration and Translation," Library Quarterly 72 (2002): 102.

9. John Budd, "A Citation Study of American Literature: Implications for Collection Management," Collection Management 8, no. 2 (1986): 56.

10. To be exact, the percentages for the twenty-year period are: for Early English, $38.6 \%$ of the total number of journal citations for this period fall within this span; for Middle English, $47.5 \%$; for Renaissance, 62.7\%; for Restoration, $63.8 \%$; for Augustan-Jacobean, $64.6 \%$; for Romantic, $61.7 \%$; for Victorian, $53.0 \%$; for Transition, $48.8 \%$; for $1919-1945,75.6 \%$; for $1946-2002,72.0 \%$; for literary theory and history, $75.8 \%$.

11. Colonial, $51.6 \%$ of the journal citations were to journal articles published in the most recent twenty years; pre-Civil War, 47.3\%; 1861-1918, 52.9\%; 1919-1945, 59.8\%; 1946-2002, 72.1\%; and theory, $81.9 \%$.

12. Heinzkill, "Characteristics of References," 353.

13. In truth, it has not been thirty years since a citation study has been done of American literature. Because my article (1980) did not cover American literature, other studies of American literature are used for comparison in this article.

14. Budd, "Characteristics of Written Scholarship," 200.

15. Stern, "Characteristics of the Literature," 203.

16. Ibid, 207.

17. Broadus, "Information Needs," 120.

18. Ibid., 125.

19. Anyone wishing to pursue a comparison of percentages in Library of Congress numbers as given by Broadus and the one in table 5 should be aware that, because Broadus includes the classification numbers for literature (i.e., PN, PR, and PS), his percentages would have to be recalculated to be anywhere near being comparable to those in table 5 . If the literary numbers are removed from his table, the percentages he gives on page 122 will show an increase.

20. Joseph C. Shipman, “General Science," Library Trends 15, no. 4 (1967): 80.

21. Rosalind Walcott, "Characteristics of Citations in Geoscience Doctoral Dissertations Accepted at United States Academic Institutions 1981-1985," Science and Technology Libraries 12, no. 2 (1991): 6.

22. Rosalind Walcott, "Characteristics of Citations in 1993 Volumes of Auk, Condor, Ibis and Wilson Bulletin," Science and Technology Libraries 15, no. 4 (1996): 31.

23. Claudia Lascar and Lorn Mendelsohn, "An Analysis of Journal Use by Structural Biologists with Applications for Journal Collection Development Decisions," College \& Research Libraries 62 (2001): 426.

24. Ibid., $426-27$.

25. Palmer and Neumann, "Information Work," 110.

26. A reviewer in TLS has expressed the same thought: "The contributors to Anniversary essays on Johnson's 'Dictionary' seldom cite electronic sources; all the same I bet they are using them regularly." H. J. Jackson, "Big and Little Matters," TLS 5354 (Nov. 11, 2005): 4.

27. One of the more recent reaffirmations of the importance of browsing is: "Humanists continue to follow familiar patterns ... browsing is widely practiced." Palmer and Neumann, "Information Work," 89.

28. "Their scanning mode of reading pushes the boundaries as they regularly browse leads in areas outside their expertise." Palmer and Neumann, "Information Work," 102, and Broadus, "Information Needs," 124. The percentage that Broadus came up with for titles outside of the humanities is $43.4 \%$. However, these are items requested to be read by his group of humanists at a research center and are, therefore, not necessarily cited in their final published products.

29. Palmer and Neumann, "Information Work," 86.

30. Giles Gunn, "Interdisciplinary Studies," in Introduction to Scholarship in Modern Languages and Literatures, ed. Joseph Gibaldi, 2nd ed. (New York: Modern Language Association of America, 1992): 239-61.

31. Palmer and Neumann detail the efforts of the Modern Language Association through the sponsorship of various monographs that show the relation of literary studies to other fields. These monographs often use the term "interdisciplinary" to show the interest of literary scholars in other areas; however, lately, "interdisciplinary" is undergoing redefinition by Gunn and others. Palmer and Neumann, "Information Work," 89.

32. Palmer and Neumann, "Information Work," 102. 


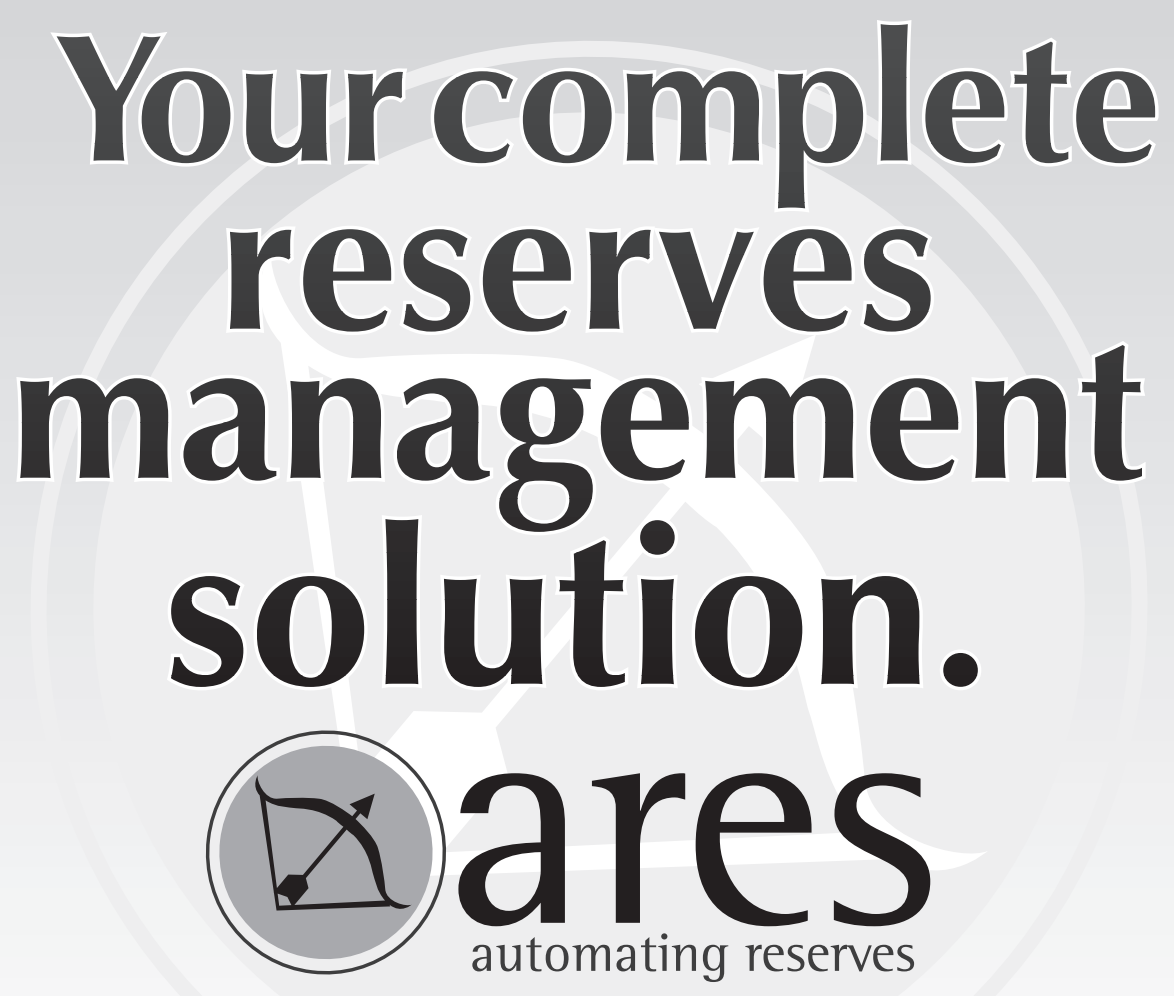

Ares is the reserve management software from the company that brought you OCLC ILLiad-the market leader in ILL management software.

Key Features:

- Web based faculty and student interface allows 24/7 access to electronic reserves

- Support and management of both electronic and hard copy reserve items

- Extensive copyright management functionality including integration with the CCC rights management database and direct to publisher requesting

See a Demo in Booth 153 at the ACRL National Conference!

Ares is now available directly from most OCLC Regional Service Providers.
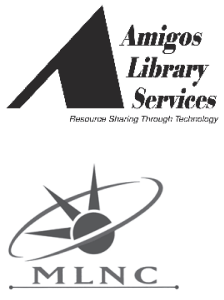

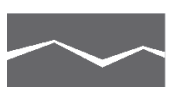

BCR blbllographlcal
center for research

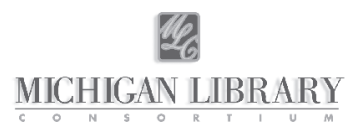

$$
\text { nelinet Nylink }
$$

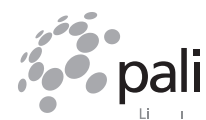

\section{Sol iNET \\ WiLS \\ A NETWORK OF KNOWLEDGE}

